\title{
Veterinary World reviewer acknowledgment 2021
}

\author{
A. V. Sherasiya and Nazir
}

Veterinary World, Star, Gulshan Park, NH-8A, Chandrapur Road, Wankaner - 363621, Dist. Morbi, Gujarat, India.

Corresponding author: A. V. Sherasiya, e-mail: editorveterinaryworld@gmail.com

Nazir: veterinaryworldpublisher@gmail.com

Received: 21-02-2022, Published online: 07-03-2022

doi: www.doi.org/10.14202/vetworld.2022.531-536 How to cite this article: Sherasiya AV, Nazir (2022) Veterinary World reviewer acknowledgment 2021, Veterinary World, 15(3): 531-536.

\section{Contributing reviewers}

Veterinary World editorial team would sincerely like to thank all of our reviewers who contributed to peer review for the journal in 2021.

\begin{tabular}{|c|c|c|c|}
\hline No. & Reviewer & Country & Reviewed articles \\
\hline 1 & A. Chwalibog & Denmark & 3 \\
\hline 2 & A. I. Paştiu & Romania & 2 \\
\hline 3 & A. R. Silva & Brazil & 2 \\
\hline 4 & A. Towhidi & Iran & 1 \\
\hline 6 & Abdelfattah H Eladl & Egypt & 1 \\
\hline 7 & Abel Villa-Mancera & Mexico & 1 \\
\hline 8 & Abid Aslam Maan & Pakistan & 1 \\
\hline 9 & Abolghasem Siyadatpanah & Iran & 1 \\
\hline 10 & Adeyinka Jeremy Adedeji & Nigeria & 1 \\
\hline 11 & Adolfo PazSilva & Spain & 1 \\
\hline 13 & Ahmed Ali Mahmoud Saleh & Japan & 2 \\
\hline 14 & Ahmed S. Abdoon & Egypt & 3 \\
\hline 15 & Ahrar Khan & Pakistan & 1 \\
\hline 16 & Ailian Geng & China & 1 \\
\hline 17 & Alejandro Hidalgo & Chile & 3 \\
\hline 18 & Alejo Menchaca & Uruguay & 1 \\
\hline 19 & Alfredo Medrano & Mexico & 2 \\
\hline 20 & Amira Taman & Egypt & 1 \\
\hline 21 & Ammar Ahmad Khan & Pakistan & 3 \\
\hline 22 & Amy C Murillo & USA & 1 \\
\hline 23 & Andrea L. Looney & USA & 1 \\
\hline 24 & Andrew A. Butler & USA & 1 \\
\hline 25 & Anihouvi H. Anihouvi & Belgium & 1 \\
\hline 26 & Ankit Magotra & India & 1 \\
\hline 27 & Anna Stanicka & Poland & 1 \\
\hline 29 & Antonello Santini & Italy & 1 \\
\hline 30 & Antonio Bevilacqua & Italy & 1 \\
\hline 33 & Archana Jain & India & 3 \\
\hline 35 & Arda Sozcu & Turkey & 2 \\
\hline 36 & Arda Yildirim & Turkey & 2 \\
\hline 37 & Ariadna Flores Ortega & Mexico & 2 \\
\hline 39 & Ariel C. Toloza & Argentina & 1 \\
\hline 40 & Arockiasamy Arun Prince Milton & India & 1 \\
\hline 41 & Ashok Dangi & India & 1 \\
\hline 43 & Ashwani Kumar & India & 2 \\
\hline 44 & Ayman Abdel-Aziz Swelum & Egypt & 3 \\
\hline 45 & B. Hoffmann & Germany & 1 \\
\hline 46 & Bahador Sarkari & Iran & 2 \\
\hline 47 & Bambang Pontjo Priosoeryanto & Indonesia & 1 \\
\hline 48 & Batoul Mohamed Izzularab & Egypt & 2 \\
\hline
\end{tabular}

Copyright: Sherasiya and Nazir. Open Access. This article is distributed under the terms of the Creative Commons Attribution 4.0 International License (http://creativecommons.org/licenses/by/4.0/), which permits unrestricted use, distribution, and reproduction in any medium, provided you give appropriate credit to the original author(s) and the source, provide a link to the Creative Commons license, and indicate if changes were made. The Creative Commons Public Domain Dedication waiver (http://creativecommons.org/publicdomain/zero/1.0/) applies to the data made available in this article, unless otherwise stated. 


\begin{tabular}{|c|c|c|c|}
\hline No. & Reviewer & Country & Reviewed articles \\
\hline 49 & Belgin Siriken & Turkey & 2 \\
\hline 50 & Bernard Davoust & France & 1 \\
\hline 51 & Breda Jakovac-Strajn & Slovenia & 1 \\
\hline 52 & Brian D. Nielsen & USA & 1 \\
\hline 54 & Buket Er Demirhan & Turkey & 2 \\
\hline 55 & Bulent Ekiz & Turkey & 1 \\
\hline 56 & C. Del Prete & Italy & 2 \\
\hline 57 & C. M. Franco & Spain & 1 \\
\hline 58 & Carlos Landaeta-Aqueveque & Chile & 1 \\
\hline 59 & Chao-Nan Lin & Taiwan & 1 \\
\hline 60 & Charleata A Carter & USA & 2 \\
\hline 61 & Charoonluk Jirapattharasate & Thailand & 1 \\
\hline 62 & Chongling Yan & China & 1 \\
\hline 63 & Claude T Sabeta & South Africa & 1 \\
\hline 64 & Claudio Genchi & Italy & 2 \\
\hline 65 & Cord M. Brundage & USA & 1 \\
\hline 66 & Dakshina Bisht & India & 1 \\
\hline 67 & Dalia Hamza & Egypt & 1 \\
\hline 68 & Danielle Bastos Araujo & Brazil & 1 \\
\hline 69 & David Gleeson & Ireland & 1 \\
\hline 70 & Davinson Chuka Anyogu & Nigeria & 1 \\
\hline 71 & Deepa PM & India & 1 \\
\hline 72 & Deepan Kishore & USA & 2 \\
\hline 73 & Dhanush Krishna Balakrishnannair & India & 2 \\
\hline 74 & Diana Riebold & Germany & 1 \\
\hline 76 & Dmitriy V. Volokhov & USA & 2 \\
\hline 77 & Domenico Galante & Italy & 1 \\
\hline 78 & Domingo Fernández García & Spain & 1 \\
\hline 79 & Dongwan Yoo & USA & 1 \\
\hline 80 & Elina B. Reinoso & Argentina & 1 \\
\hline 81 & Eloiza Teles Caldart & Brazil & 1 \\
\hline 82 & Elshymaa A. Abdelnaby & Egypt & 1 \\
\hline 83 & Emily A. Smith & USA & 1 \\
\hline 84 & Enrica Zucca & Italy & 1 \\
\hline 85 & Erika van Zyl & South Africa & 2 \\
\hline 86 & Erma Safitri & Indonesia & 2 \\
\hline 87 & Esther Kamau & Kenya & 1 \\
\hline 88 & F. Pandolfi & UK & 1 \\
\hline 89 & Fabrizio Bertelloni & Italy & 3 \\
\hline 90 & Farida Khammar & Algeria & 2 \\
\hline 91 & Fazul Nabi & Pakistan & 1 \\
\hline 92 & Felipe da Silva Krawczak & Brazil & 1 \\
\hline 94 & Francesca Maria Sarti & Italy & 1 \\
\hline 95 & Francesco Mira & Italy & 1 \\
\hline 96 & Françoise Leriche & France & 1 \\
\hline 97 & Giuseppe Bertoni & Switzerland & 2 \\
\hline 98 & Glayciane Costa Gois & Brazil & 1 \\
\hline 100 & Gosia Zobel & New Zealand & 1 \\
\hline 102 & Halit imik & Turkey & 2 \\
\hline 103 & Hany Mhany Ragab AbdelLatif & Egypt & 1 \\
\hline 104 & Harish Padh & India & 1 \\
\hline 105 & Harvie P. Portugaliza & Philippines & 3 \\
\hline 106 & Hasria Alang & Indonesia & 2 \\
\hline 107 & Hassan Vatandoost & Iran & 1 \\
\hline 108 & Hazem Shaheen & Egypt & 1 \\
\hline 109 & Heba Ahmed Hussein Ahmed & Egypt & 1 \\
\hline 110 & Heriberto Caballero-Ortega & Mexico & 1 \\
\hline 111 & HuaJi Qiu & China & 2 \\
\hline 112 & Huseyin Yilmaz & Turkey & 1 \\
\hline 113 & Hussam Mohamed Mohamed Ibrahim & Egypt & 1 \\
\hline 114 & Hussein Awad Hussein & Egypt & 1 \\
\hline
\end{tabular}




\begin{tabular}{|c|c|c|c|}
\hline No. & Reviewer & Country & Reviewed articles \\
\hline 115 & Hyunjin Shin & Korea & 1 \\
\hline 116 & Ibrahim Adisa Raufu & Nigeria & 1 \\
\hline 117 & Igor Yankin & USA & 1 \\
\hline 118 & Indrasen Chauhan & India & 3 \\
\hline 119 & Inga Stadaliene & Lithuania & 2 \\
\hline 120 & Ioana Matei & Romania & 1 \\
\hline 121 & Ipsita Mohanty & USA & 5 \\
\hline 122 & Ismail Hakkı Tekiner & Turkey & 2 \\
\hline 123 & J Efren Ramirez-Bribiesca & Mexico & 1 \\
\hline 124 & J. P. Vico & Argentina & 2 \\
\hline 125 & Jareerat Aiemsaard & Thailand & 1 \\
\hline 126 & Jaruwan Khonmee & Thailand & 1 \\
\hline 127 & Jerome Andonissamy & India & 1 \\
\hline 128 & Jesus Alonso PantiMay & Mexico & 1 \\
\hline 129 & Ji Bao & China & 1 \\
\hline 130 & Jitender Dubey & USA & 1 \\
\hline 131 & Josef illek & Czech Republic & 3 \\
\hline 132 & Joseph G. Barnes & USA & 1 \\
\hline 133 & Joshua Mbanga & Zimbabwe & 1 \\
\hline 134 & Kalyan Sundar Das & India & 1 \\
\hline 135 & Kannan Thandavan Arthanari & India & 1 \\
\hline 136 & Kate Fenner & Australia & 1 \\
\hline 137 & Katrin StrutzbergMinder & Germany & 1 \\
\hline 138 & Kaushal Kishor Rajak & India & 1 \\
\hline 139 & Kavous Solhjoo & Iran & 1 \\
\hline 140 & Keita Iyori & Japan & 1 \\
\hline 141 & kekungu puro & India & 1 \\
\hline 142 & Keshore R. Bidasee & USA & 1 \\
\hline 143 & Khan Sharun & India & 1 \\
\hline 144 & Khawla Elati & Tunisia & 1 \\
\hline 145 & Kosta Y. Mumcuoglu & Israel & 1 \\
\hline 146 & Koushlesh Ranjan & India & 1 \\
\hline 147 & Laxmi Narayan Sarangi & India & 1 \\
\hline 148 & Lena Sizova & Russia & 1 \\
\hline 149 & Lenin Lenin Aguirre Riofrio & Ecuador & 1 \\
\hline 150 & Liben Chen & USA & 1 \\
\hline 151 & Liga Kovalcuka & Latvia & 2 \\
\hline 152 & Lin Jiang & China & 1 \\
\hline 153 & Linous Munsimbwe & Zambia & 2 \\
\hline 154 & Linsen Zan & China & 1 \\
\hline 156 & Lucía Calleros & Uruguay & 1 \\
\hline 157 & Luciana Catalina & Romania & 1 \\
\hline 159 & Luis Nestor Apaza Ticona & Spain & 1 \\
\hline 160 & Luis Tello & USA & 1 \\
\hline 162 & Luisa De Martino & Italy & 1 \\
\hline 163 & Luit Barkalita & Belgium & 1 \\
\hline 164 & Lukasz Migdal & Poland & 2 \\
\hline 165 & M. Duggan & UK & 1 \\
\hline 166 & M. Teresa Sancho & Spain & 1 \\
\hline 167 & M. Zamri Saad & Malaysia & 3 \\
\hline 168 & Mabel Kamweli Aworh & Nigeria & 2 \\
\hline 169 & Magdalena Zając & Poland & 2 \\
\hline 170 & Mahmoud Mohey Elhaig & Egypt & 1 \\
\hline 171 & Mahreen UI Hassn & UK & 1 \\
\hline 172 & Maja Josip Velhner & Serbia & 2 \\
\hline 173 & Manuela Oliveira & Portugal & 1 \\
\hline 174 & Marc Drillich & Austria & 1 \\
\hline 175 & Maria Cristina Cozzi & Italy & 1 \\
\hline 176 & Maria Fernanda Castro Burbarelli & Brazil & 1 \\
\hline 177 & Maria Luisa Dettori & Italy & 1 \\
\hline 178 & Mariana Malzoni Furtado & Brazil & 1 \\
\hline 179 & Mariano E. Fernandez-Miyakawa & Argentina & 1 \\
\hline
\end{tabular}




\begin{tabular}{|c|c|c|c|}
\hline No. & Reviewer & Country & Reviewed articles \\
\hline 180 & Mário Manuel Dinis Ginja & Portugal & 2 \\
\hline 181 & Marta Vascellari & Italy & 1 \\
\hline 182 & Maryam Azizkhani & Iran & 1 \\
\hline 183 & Maryam Dadar & Iran & 1 \\
\hline 184 & Massimo Vignoli & Italy & 1 \\
\hline 185 & Md Samun Sarker & Bangladesh & 1 \\
\hline 186 & Md. Ahaduzzaman & Bangladesh & 2 \\
\hline 187 & Md. Golzar Hossain & Bangladesh & 1 \\
\hline 188 & Md. Sadequl Islam & Bangladesh & 1 \\
\hline 189 & Mehmet Gultekin & Turkey & 1 \\
\hline 190 & Michelle Moore & USA & 1 \\
\hline 191 & Miguel Angel Barrera Silva & Mexico & 1 \\
\hline 192 & Miguel Angel Garcia-Bereguiain & Ecuador & 1 \\
\hline 193 & Miguel Quaresma & Portugal & 2 \\
\hline 194 & Min Yue & China & 2 \\
\hline 195 & Mirosław Mariusz Michalski & Poland & 1 \\
\hline 196 & Mohamed Abdo Rizk & Japan & 1 \\
\hline 197 & Mohamed E. Abd & Egypt & 1 \\
\hline 198 & Mohamed G. Hedia & Egypt & 1 \\
\hline 199 & Mohamed Hamed & Egypt & 1 \\
\hline 200 & Mohamed S. Kamel & Egypt & 1 \\
\hline 204 & Mohamed Salah Ayyat & Egypt & 2 \\
\hline 205 & Mohammad Abdul Momin Siddique & Vietnam & 1 \\
\hline 206 & Mohammad Borhan Alzghoul & Jordan & 1 \\
\hline 207 & Mohammad Moniruzzaman & Bangladesh & 1 \\
\hline 210 & Mohammad Zahangir Alam & Bangladesh & 1 \\
\hline 211 & Mohammed A. E. & Egypt & 3 \\
\hline 212 & Mohammed El-Houadfi & Morocco & 1 \\
\hline 213 & Mohammed Fouad El Basuini & Egypt & 2 \\
\hline 214 & Mohammed Manosur & Sudan & 1 \\
\hline 215 & Mohammed S. ALhajj & Saudi Arabia & 1 \\
\hline 216 & Mohan Singh Thakur & India & 2 \\
\hline 217 & Monthon Lertcanawanichakul & Thailand & 1 \\
\hline 218 & Mostafa F.N. Abushahba & USA & 2 \\
\hline 219 & Moutaz Zarkawi & Syria & 1 \\
\hline 220 & Muhammad Irfan-ur-Rehman Khan & Pakistan & 2 \\
\hline 221 & Murat Sevik & Turkey & 2 \\
\hline 222 & Nadeem Shabir & India & 1 \\
\hline 223 & Natalia Kasica & Poland & 1 \\
\hline 224 & Nathalia Maria del Pilar Correa Valencia & Colombia & 2 \\
\hline 225 & Naveen Kondru & USA & 2 \\
\hline 226 & Nazli Ercan & Turkey & 1 \\
\hline 227 & Nesrein M. Hashem & Egypt & 1 \\
\hline 228 & Olfat Anter Mahdy & Egypt & 1 \\
\hline 229 & P.S. Erickson & USA & 1 \\
\hline 230 & Padet Siriyasatien & Thailand & 1 \\
\hline 231 & Paiboon Sithithaworn & Thailand & 1 \\
\hline 232 & Pankaj Kumar & India & 1 \\
\hline 233 & Paola Modesto & Italy & 1 \\
\hline 234 & Paramanandham Krishnamoorthy & India & 1 \\
\hline 235 & Patricia Escandón & Colombia & 1 \\
\hline 236 & Peerapol Sukon & Thailand & 1 \\
\hline 237 & Pengfei Li & China & 1 \\
\hline 238 & Phillip J Rincker & USA & 1 \\
\hline 239 & Pie Ntampaka & Rwanda & 2 \\
\hline 240 & Pınar Peker Akalın & Turkey & 2 \\
\hline 241 & Pınar Şanlıbaba & Turkey & 2 \\
\hline 242 & Piotr Szweda & Poland & 1 \\
\hline 243 & Pourouchottamane R & India & 1 \\
\hline 244 & Prabhat Kumar Pankaj & India & 1 \\
\hline 245 & Prapansak Srisapoome & Thailand & 1 \\
\hline
\end{tabular}




\begin{tabular}{|c|c|c|c|}
\hline No. & Reviewer & Country & Reviewed articles \\
\hline 246 & Qingping Wu & China & 2 \\
\hline 247 & R.Umaya Suganthi & India & 2 \\
\hline 248 & Rafiqul Islam & India & 1 \\
\hline 249 & Rajender Kumar & India & 1 \\
\hline 250 & Rajib Deb & India & 1 \\
\hline 251 & Randa Ismail Alarousy & Egypt & 2 \\
\hline 252 & Randall Lockwood & USA & 1 \\
\hline 253 & Raquel Salazar Lugo & Venezuela & 1 \\
\hline 254 & Reginaldo Nassar Ferreira & Brazil & 1 \\
\hline 255 & Remil L. Galay & Philippines & 1 \\
\hline 256 & Richard Churchchil & India & 1 \\
\hline 257 & Rob Armstrong & USA & 1 \\
\hline 258 & ROBERT L. SCHARFF & USA & 2 \\
\hline 259 & Rolf karl Schuster & United Arab Emirates & 1 \\
\hline 260 & Rosa Estela Quiroz Castaeda & Mexico & 1 \\
\hline 261 & Ruben Barajas Cruz & Mexico & 1 \\
\hline 262 & S. B. N. Rao & India & 1 \\
\hline 263 & S. Gnat & Poland & 2 \\
\hline 264 & S.K. Mondal & India & 1 \\
\hline 265 & Saber Esmaeili & Iran & 2 \\
\hline 266 & Sabrina Locatelli & France & 1 \\
\hline 267 & Sabrina Sheila Greening & New Zealand & 1 \\
\hline 268 & Saeed Alamian & Iran & 1 \\
\hline 269 & Sahadeb Dey & India & 1 \\
\hline 270 & Sahar Rihan Fadhel & Iraq & 1 \\
\hline 271 & Salma Bessalah & Tunisia & 2 \\
\hline 272 & Sana A M Elgayar & Egypt & 1 \\
\hline 273 & Sandeep Ghatak & India & 1 \\
\hline 274 & Sandeep Kaswan & India & 1 \\
\hline 275 & Saurabh Kadyan & India & 1 \\
\hline 276 & Sayed Haidar Abbas & China & 1 \\
\hline 277 & Semíramis Guimarães & Brazil & 1 \\
\hline 278 & Senol Celik & Turkey & 2 \\
\hline 279 & Serkan Irfan Kose & Turkey & 1 \\
\hline 280 & Shabnoor Iqbal & Pakistan & 1 \\
\hline 281 & Shaimaa Ali Selim & Egypt & 1 \\
\hline 283 & Shannon K French & Canada & 1 \\
\hline 284 & Sheeva Bhattarai & Denmark & 1 \\
\hline 285 & Shekhar Ramchandra Bhagwat & India & 1 \\
\hline 286 & Shih-Ching Yen & Taiwan & 1 \\
\hline 287 & Simona Martinotti & Italy & 1 \\
\hline 288 & Sina Salajegheh Tazerji & Iran & 2 \\
\hline 289 & Sintija Jonova & Latvia & 1 \\
\hline 290 & Socorro Retana-Marquez & Mexico & 4 \\
\hline 291 & Somwang Lekjing & Thailand & 1 \\
\hline 292 & Song Gao & China & 1 \\
\hline 294 & Sorin Daniel Dan & Romania & 1 \\
\hline 296 & Spenser J. Babb-Biernacki & USA & 1 \\
\hline 297 & Sreenu Makkena & India & 1 \\
\hline 298 & Stephania Madrid Gaviria & Colombia & 1 \\
\hline 299 & Süleyman Cilek & Turkey & 4 \\
\hline 300 & sunday charles olaogun & Nigeria & 1 \\
\hline 301 & Susana Astiz & Spain & 2 \\
\hline 302 & Tanko Polycarp Nwunuji & Nigeria & 1 \\
\hline 303 & Tapas Kumar Sar & India & 1 \\
\hline 304 & Tariq Jamil & Germany & 2 \\
\hline 305 & tequiero abuom okumu & Kenya & 1 \\
\hline 306 & Tetiana BAKHUR & Ukraine & 1 \\
\hline 307 & V. C. RAYULU & India & 1 \\
\hline 308 & V. Di Marco & Italy & 1 \\
\hline 309 & Vanessa S. Cruz & Brazil & 5 \\
\hline 310 & Varij Nayan & India & 2 \\
\hline
\end{tabular}




\begin{tabular}{|c|c|c|c|}
\hline No. & Reviewer & Country & Reviewed articles \\
\hline 311 & Ved Prakash & India & 1 \\
\hline 312 & Venkateswara rao Parimisetti & India & 1 \\
\hline 313 & Vibuntita Chankitisakul & Thailand & 1 \\
\hline 314 & Vijay J. Jadhav & India & 1 \\
\hline 315 & Vijaya Kumar Anumolu & India & 1 \\
\hline 316 & Vincenzo Tufarrelli & Italy & 1 \\
\hline 317 & Vishal S Suthar & India & 2 \\
\hline 318 & Wenliang Li & China & 1 \\
\hline 319 & Widya Paramita Lokapirnasari & Indonesia & 4 \\
\hline 320 & William Alberto CanFranco & Colombia & 1 \\
\hline 321 & William J. Fielding & Bahamas & 1 \\
\hline 322 & Win Surachetpong & Thailand & 2 \\
\hline 323 & Wojciech Kozdruń & Poland & 1 \\
\hline 324 & Wojciech Zygner & Poland & 3 \\
\hline 325 & Xue Gao & China & 1 \\
\hline 326 & Ya-Ching Lin & Taiwan & 2 \\
\hline 327 & Yacine Titouche & Algeria & 2 \\
\hline 328 & Yafeng Huang & China & 1 \\
\hline 329 & Yaron Dekel & Israel & 1 \\
\hline 330 & Yaser Hamadeh Tarazi & Jordan & 1 \\
\hline 331 & Yasser M. Albadrany & Iraq & 1 \\
\hline 332 & Yoon Kong & South Korea & 1 \\
\hline 333 & Yosra AMdouni & Tunisia & 2 \\
\hline 334 & Yosra Soltan & Egypt & 1 \\
\hline 335 & Youssef A. Attia & Saudi Arabia & 1 \\
\hline 336 & Yukita Sato & Japan & 1 \\
\hline 337 & Yves Millemann & France & 1 \\
\hline 338 & Zaven Karalyan & Armenia & 2 \\
\hline
\end{tabular}

$* * * * * * * *$ 